\title{
La restauración de la tapia monumental: pasado, presente y futuro
}

\section{Monumental rammed earth conservation: past, present and future}

C. Mileto ${ }^{(*)}$, F. Vegas López-Manzanares ${ }^{(*)}, \underline{\text { L. García-Soriano }}^{(*)}$

\section{RESUMEN}

El creciente interés por el estudio de la arquitectura de tierra que se desarrolló en Europa a finales de los años setenta, germinó también aunque de manera más tardía en España. A la par del creciente interés por estas estructuras históricas de tierra, se incrementó también el número de proyectos de restauración llevados a cabo en estos edificios. A partir de la década de 1980, las intervenciones en arquitectura de tapia en toda la Península Ibérica fueron en aumento, por lo que estas actuaciones tienen también ya un cierto recorrido. Esta investigación analiza las intervenciones realizadas en edificios monumentales de tapia en el territorio español financiadas con fondos estales, con las lesiones afrontadas más frecuentes, los criterios de intervención adoptados en cada caso, las técnicas constructivas empleadas y los resultados obtenidos.

Palabras clave: restauración, arquitectura de tierra, tapia, criterios

\section{ABSTRACT}

The growing interest in the study of earthen architecture developed in Europe in the late 1970s reached Spain at a later stage. As the interest in these historical earthen architecture structures grew, so did the number of restoration projects for monumental buildings originally constructed using the technique of rammed earth. As the interventions on earthen architecture in the Iberian Peninsula increased in the 1980s, these restorations are now relatively old. This research aims to have an overview the interventions carried out on rammed earth monumental buildings in Spain using national funds, through a global analysis of the most frequent pathologies found in these structures, the criteria adopted, the constructive techniques used, as well as the results obtained.

Keywords: restoration, earthen architecture, rammed earth, criteria.

(*) Universitat Politècnica de València, (España)

Persona de contacto/Corresponding author: ligarso@arq.upv.es (L. García-Soriano)

ORCID: http://orcid.org/oooo-0oo2-6987-8802 (C. Mileto); http://orcid.org/oooo-0oo3-0315-6839 (F. Vegas); http://orcid.org/oooo-0001-9696-7045 (L. García-Soriano)

Cómo citar este artículo/Citation: Mileto, C., Vegas López-Manzanares, F., García-Soriano, L. (2017). La restauración de la tapia monumental: pasado, presente y futuro. Informes de la Construcción, 69(548): e231, doi: http://dx.doi.org/10.3989/ic.16.160.

Copyright: (C) 2017 CSIC. Licencia / License: Salvo indicación contraria, todos los contenidos de la edición electrónica de Informes de la Construcción se distribuyen bajo una licencia de uso y distribución Creative Commons Attribution License (CC BY) Spain 3.o. 


\section{INTRODUCCIÓN}

La arquitectura de tapia ha formado parte de la cultura de los materiales de construcción en la Península Ibérica y posee un gran valor tanto por su origen como por el grado de conservación de estas estructuras y su adaptación con el medio ambiente (1). La construcción con tapia en todas sus variantes se ha desarrollado en España a lo largo de la historia tanto en la arquitectura monumental como en la tradicional y vernácula hasta que, a principios del siglo pasado, entró progresivamente en declive, llegando prácticamente a desaparecer. Este fenómeno puede atribuirse principalmente al creciente desarrollo industrial y a la introducción de nuevos materiales de construcción que fueron sustituyendo los sistemas constructivos tradicionales de tierra, por considerarlos de peores prestaciones técnicas y, en definitiva, obsoletos. Sin embargo, a partir de las últimas décadas del siglo XX empieza a aumentar el interés por la arquitectura de tierra que comienza a estudiarse progresivamente (2). Por otro lado, las intervenciones en edificios históricos de tapia también poseen ya cierto recorrido. Estas intervenciones se caracterizan por la diversidad de criterios, materiales y técnicas empleadas que también se ha reflejado en los resultados obtenidos con estas intervenciones a medio y largo plazo.

La inmensa variedad de criterios y alternativas abre un gran abanico de posibilidades en las soluciones propuestas. Además, cada intervención puede generar diversos tipos de consecuencias o resultados con el paso del tiempo, tanto por el envejecimiento debido al material y la técnica empleados, como por el impacto estético, estructural, etc. que genera la nueva aportación (3).

Así pues, esta investigación ha tenido como objetivo principal realizar una puesta en común de las intervenciones de restauración del patrimonio monumental de tapia, llevadas a cabo en nuestro territorio durante los últimos treinta años para poder aprender de ellas y extraer conclusiones y perspectivas para el futuro. El análisis detallado de las obras seleccionadas, la puesta en común de las distintas experiencias, la reflexión sobre las técnicas, criterios de intervención y resultados obtenidos, y el conocimiento derivado, tanto a nivel teórico como técnico de este proceso de revisión de intervenciones, garantizan el interés de la investigación.

\section{METODOLOGÍA DE INVESTIGACIÓN}

La metodología de investigación está basada en el análisis pormenorizado de casos de estudio usando métodos cualitativos sobre la base de una amplia información adquirida a través de diversas fuentes: primarias (entrevistas e información proporcionada directamente por los agentes implicados en la restauración del edificio) y secundarias directas (visitas técnicas a los propios edificios restaurados) e indirectas (bibliografía, documentación de archivo, proyectos, etc.), obteniendo así una aproximación metodológica múltiple (4).

\subsection{Recopilación de la información}

Se ha elaborado una base de datos con un listado lo más completo posible de obras realizadas en los últimos treinta años; se ha realizado una selección de casos de interés; y se ha recopilado la mayor cantidad de información posible sobre estos casos, consultando proyectos, expedientes y documentación existente, visitando a los edificios; etc. En esta primera fase se ha elaborado una base de datos a partir de la búsqueda e investigación exhaustiva en tres archivos (Archivo General del IPCE del Ministerio de Cultura, Archivo del Programa del 1\% Cultural del Ministerio de Fomento y Archivo Central de la Consejería de Cultura de la Junta de Andalucía), estableciendo una ficha para la catalogación completa de las obras. Los campos que contiene la ficha se dividen en cinco apartados: datos generales del edificio, datos generales de la intervención, técnicas de intervención empleadas, criterios de intervención y estado actual del edificio. Se ha recopilado información de 168 edificios monumentales distintos que corresponden a un total de 201 intervenciones inventariadas en la base de datos. La elección de estos archivos como fuente de información ha respondido a varios factores. Los dos archivos estatales custodian los expedientes de intervenciones llevadas a cabo desde la administración central: en el archivo del IPCE se encuentran las intervenciones más antiguas correspondientes a la primera mitad de los años 8o, y en el archivo del $1 \%$ Cultural, las intervenciones más recientes, desde el año 2004 en adelante. Por ello, para obtener información relativa a las intervenciones realizadas tras el traspaso de competencias a las comunidades autónomas a mediados de los años 80 es necesario acudir a los archivos autonómicos. Se ha optado por trabajar con el archivo de la Junta de Andalucía dado el gran número de edificios monumentales de tapia que existen en esta comunidad. No obstante, sería también muy interesante poder seguir desarrollando este trabajo en el resto de comunidades autónomas.

\subsection{Análisis de los casos, reflexión y puesta en común de las experiencias}

Finalizada la fase de recopilación de la información, se ha realizado el análisis pormenorizado de los casos con una metodología basada en diversos parámetros que permite estudiar de la forma más objetiva posible el proceso de restauración y los resultados alcanzados. La ficha que se ha desarrollado para la catalogación de los casos se ha dividido y estructurado en cinco partes fundamentales:

En primer lugar, se ha analizado la técnica constructiva propia del edificio original puesto que es diferente intervenir en un tipo de tapia u en otro tanto a tenor de los problemas planteados por la propia técnica (tapia de tierra, tapia calicostrada, tapia con piedras, etc.) y por el tipo de degradación presente (erosión de la superficie, desprendimiento o pérdida de la costra, lagunas, etc.).

En segundo lugar, se han estudiado las lesiones del edificio antes de la restauración, puesto que el tipo de intervención está ligada al tipo de degradación más o menos profunda, estructuralmente nociva, etc. Se han agrupado las lesiones por zonas afectadas del edificio, de modo que resulte más fácil relacionarlas con las intervenciones en un ulterior análisis. Según este esquema, se han identificado lesiones que afectan a las fábricas (pérdida de volumen, presencia de lagunas, etc.), la coronación (erosión y lavado, pérdida de cubrimiento, etc.), la superficie (erosión, pérdida de costra, presencia de sales, manchas, etc.) y la base (descalce en la cimentación, etc.).

En tercer lugar, se han examinado las técnicas de intervención empleadas que han debido tener en cuenta el material original y su estado de conservación. Las técnicas de intervención se han agrupado también por zonas de actuación, añadiendo un apartado de otras intervenciones adyacentes que pueden 
afectar de alguna manera a los muros de tapia (inserción de forjados, cubiertas y escaleras, etc.).

Posteriormente, se ha realizado el análisis de los criterios empleados en la intervención. La complejidad de esta tarea reside en analizar los criterios desde un punto de vista lo más objetivo posible. Se ha trabajado desde dos grandes frentes: los criterios de intervención (conservación, reintegración, reconstrucción y demolición) y los principios generales de la teoría de la restauración (conservación de la materia auténtica, mínima intervención, reversibilidad, compatibilidad material, compatibilidad estructural, durabilidad, actualidad expresiva y/o distinguibilidad, neutralidad), extraídos de diversos textos fundamentales en la definición de la teoría de la restauración contemporánea (5) (6) (7) (8).

Finalmente en la última parte de la ficha se realiza un pequeño análisis del estado actual del edificio y las eventuales degradaciones que presenta. A través de esta ficha se genera una base de datos en la que se ordenan y sistematizan todos los casos de estudio para poder realizar un análisis y reflexión global.

\subsection{Producción del corpus de conocimiento y difusión}

Tras el análisis de los casos y las conclusiones derivadas de la comparación cruzada, se ha alcanzado un conocimiento más sistemático de las experiencias de restauración de edificios monumentales de tapia, realizadas hasta la fecha en nuestro territorio (9) (Figura 1).

El posterior análisis de las técnicas constructivas y criterios empleados en las intervenciones ha requerido asimismo diferenciar los edificios analizados según su tipología constructiva y sus características arquitectónicas propias, ya que de ellas dependen en muchos casos los fenómenos de degradación presentes y por tanto, el tipo de intervención a realizar en cada una de las zonas estudiadas.

En consecuencia, los casos de estudio se han agrupado en tres familias: la arquitectura militar (castillos, alcazabas, torres, murallas...), la arquitectura religiosa (conventos, monasterios, iglesias...) y la arquitectura civil (palacios, casas...). Se ha reunido un total de 115 edificios que pertenecen al grupo de la arquitectura militar (34 murallas, 71 castillos y 13 torres, que son aproximadamente un 68,5\% del total de los casos de estudio), 32 edificios pertenecientes a la arquitectura religiosa (aproximadamente un 19\% del total) y 21 edificios en el grupo de la arquitectura civil (12,5\% del total). Esta distribución predominante de la arquitectura militar no es extraña, ya que se debe tener en cuenta que la tapia es una técnica constructiva muy presente en esta tipología de edificios de la arquitectura monumental.

En cuanto a las características constructivas, de los 168 edificios que forman la base de datos, 61 casos se engloban dentro de las variantes de tapia simple (aproximadamente un $37 \%$ del total), 33 edificios son de tapia con mampuestos (aproximadamente un 20\%), 27 de tapia calicostrada (aproximadamente un 16,5\% de la muestra) y 21 ejemplos son de tapia con machones y verdugadas de ladrillo (un 12,8\% del total). El resto (aproximadamente el 13,7\% restante) está formado por otras variantes constructivas como son: la tapia con esquinas y machones de sillería (6 edificios), la tapia con juntas reforzadas (3 edificios), la tapia con basamento de sillería (6 casos) y la mampostería encofrada (4 edificios). Esta distribución de las variantes constructivas responde fundamentalmente, como ya se había avanzado, a la tipología de cada caso de estudio. De este modo, como la tipología más presente en la muestra es la de la arquitectura militar y defensiva, las variantes constructivas más abundantes son por tanto las que se encuentran más asiduamente en este tipo de edificios. Es por tanto imprescindible conocer la variante constructiva original y sus fenómenos de degradación propios para poder proponer buenas soluciones a las mismas.

\section{ANÁLISIS DE LOS CRITERIOS DE INTERVENCIÓN}

La gran mayoría de las intervenciones analizadas cubren un amplio abanico de combinaciones de acciones de conservación, reintegración y reconstrucción con porcentajes de actuación variables entre estos tres componentes. El empleo sistematizado de los parámetros a través de las fichas ha permitido analizar cada intervención y a su vez realizar posteriormente un análisis cruzado, obteniendo con un método inductivo una serie de conclusiones generales sobre los criterios de intervención empleados.

\subsection{Evolución temporal de los criterios adoptados}

Según el análisis de los casos, se ha podido establecer que durante el primer periodo de los años 80 las intervenciones consistieron fundamentalmente en reconstrucciones más o menos generales que conferir al monumento la presencia estética de antaño, en algunos casos de forma más comedida con reconstrucciones parciales, y en otros de forma más extensiva, con reconstrucciones totales que pretendían devolver el monumento a un punto concreto de su historia que se consideraba de mayor interés arquitectónico. Se trata de unos criterios que es posible relacionar con una línea de pensamiento historicista que, aunque en muchas otras zonas de Europa en este momento ya se había abandonado casi totalmente, en España seguía muy presente hasta el final de la década. A finales de la década de los 80 y principios de los 90 empezaron a imponerse ya unos criterios más propios de la línea conservadora, realizándose un mayor número de intervenciones basadas en reconstrucciones parciales de los muros y reintegraciones de elementos faltantes. La tendencia conservadora fue ganando fuerza con el tiempo y en la década de los años 2000 continuaron primando las actuaciones de reintegración y reconstrucción parcial de volúmenes. No obstante, no se trata de líneas de actuación perfectamente marcadas y definidas, por lo que siempre es posible encontrar excepciones a este planteamiento global.

\subsection{Estrategias según la zona del muro en la que se interviene}

Las estrategias de intervención empleadas han variado también a tenor de la zona del muro afectada.

En la base de los muros. Las actuaciones en la base de los muros consisten generalmente en el recalce ocasional y el completamiento parcial de las lagunas existentes en la cimentación. El criterio predominantemente adoptado es la reconstrucción parcial de volúmenes (Gráficos A2, B2 y C2 de la 


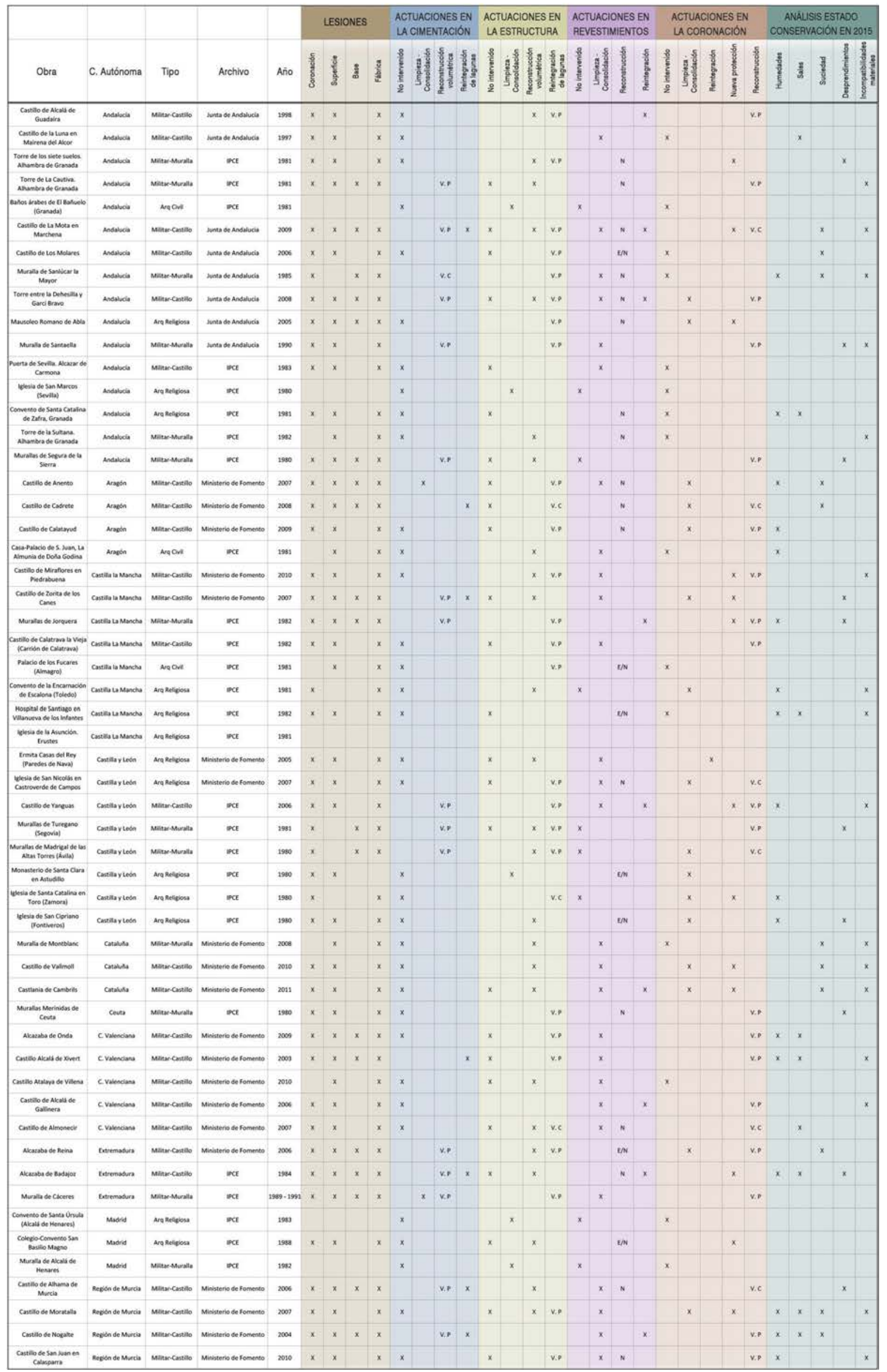

Figura 1. Tabla ejemplo de análisis cruzado de los casos de estudio. 
Figura 2), con diversas variantes en función de los materiales empleados en la reconstrucción.

En los muros. Las propuestas más antiguas reconstruían los muros total o parcialmente de forma bastante extensiva, mientras que las intervenciones más recientes plantean frecuentemente la limpieza y la reintegración de lagunas (más del 60\% de los casos), conservando los restos existentes, realizándose en ocasiones trabajos de reconstrucción pero más localizados (aproximadamente un 25\% de los casos) (Gráfico C1 de la Figura 2).

En la coronación. Las intervenciones en la coronación han asumido criterios muy distintos con la evolución temporal de las propuestas. Los proyectos más antiguos proponían mayoritariamente $(52 \%$ del total de las reconstrucciones de los años 80) la recuperación completa, reconstruyendo los muros hasta la cota original, reproduciendo incluso en muchos casos los remates y almenas, frecuentemente concentrándose en los elementos más significativos del conjunto (como por ejemplo la Torre del Homenaje), dejando sin intervenir el resto de muros y lienzos (Imagen A de la Figura 3). En muchas ocasiones estas reconstrucciones no llevaban asociados estudios previos completos, por lo que se trataba de auténticas reinterpretaciones proyectuales. Con el tiempo este criterio fue cambiando y las reconstrucciones fueron cada vez más contenidas, llegando a los proyectos más actuales en los que se tiende a sobreelevar las coronaciones lo mínimo posible, normalmente elevando un módulo de tapia que sirve de protección a los restos existentes y que se adapta a la altura de los mismos de forma escalonada (10) (Imagen D de la Figura 5).
1980-1989

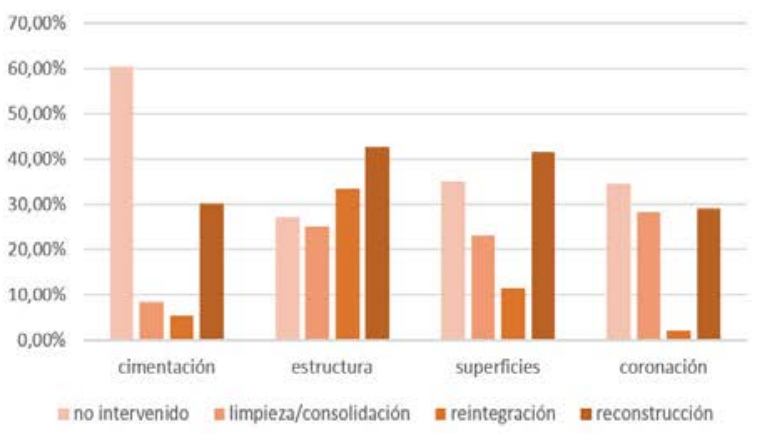

A1. Análisis de los criterios de intervención (años 80 ) según la zona del muro en la que se interviene

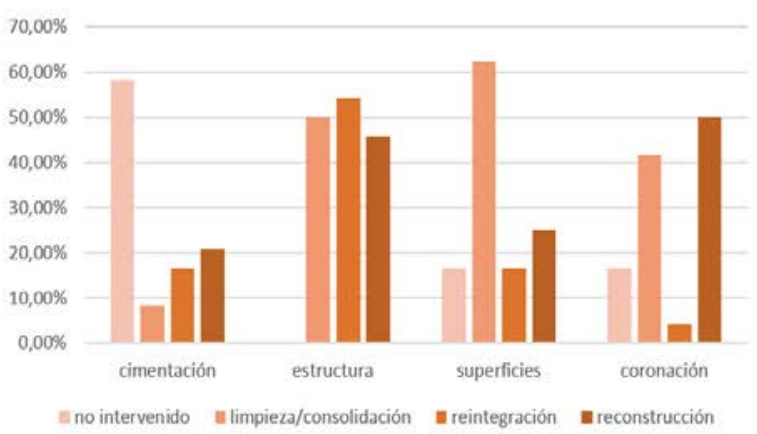

B1. Análisis de los criterios de intervención (años 90) según la zona del muro en la que se interviene

2000-2013

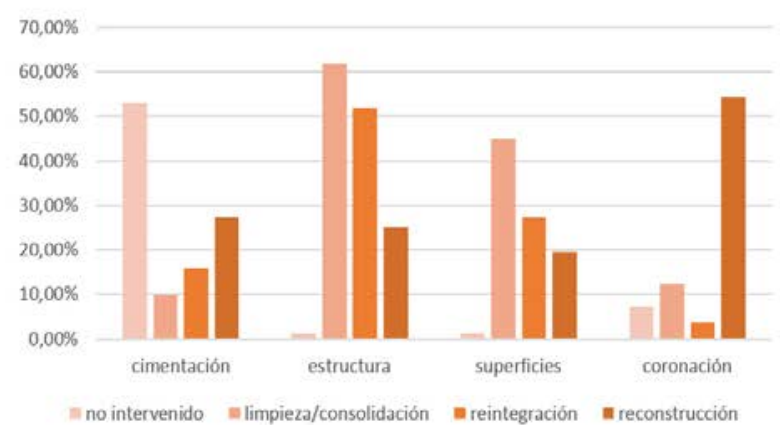

C1. Analisis de los criterios de intervención (años 2000) según la zona del muro en la que se interviene

1980-1989

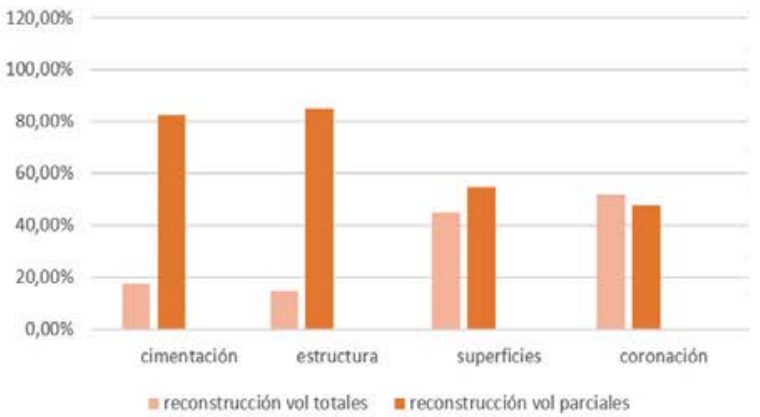

A2. Análisis del tipo de reconstrucción (años 80 ) según la zona del muro en la que se interviene

1990-1999

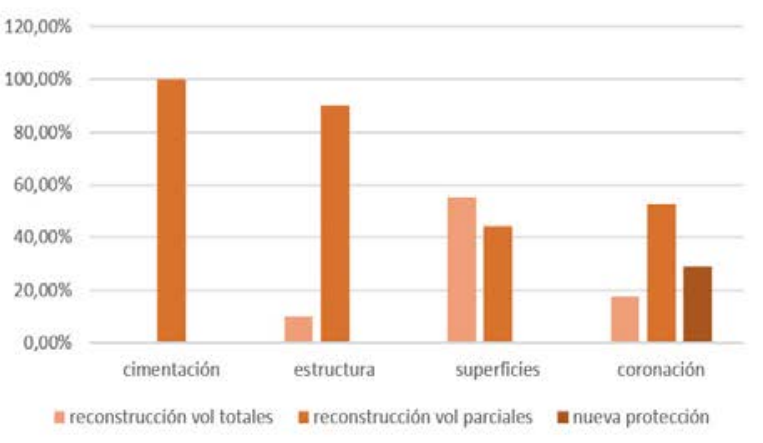

B2. Análisis del tipo de reconstrucción (años 90) según la zona del muro en la que se interviene

Figura 2. Gráficos de los resultados del análisis de los criterios de intervención en las tres décadas. 
Otra estrategia para la proteger la coronación y mantener el perfil erosionado de la misma es la inserción de un nuevo elemento de protección (un mortero...), que en la década de los 90 y años 2000 es también un recurso frecuente, encontrado en aproximadamente el 20-25\% de los casos (Gráficos B2 y C2 de la Figura 2) (Imagen C de la Figura 3).

En las superficies. Esta zona requiere unas actuaciones de tratamiento específico que no eran frecuentes en la década de los 80 , grupo en el que el $35 \%$ de las intervenciones no consideraban las superficies de forma específica. En los años 90 este porcentaje disminuye, y en los 2000 son prácticamente inexistentes las actuaciones en las que no se interviene en las superficies de las tapias (Gráficos A1, A2 y A3 de la Figura 2). En esta zona las actuaciones de demolición están normalmente presentes como acciones parciales, generalmente ligadas a la eliminación de materiales sueltos y disgregados antes de ejecutar la nueva intervención. En algunos casos se demolieron también materiales de actuaciones anteriores por considerarlos dañinos para el edificio y, en el caso de las superficies, fundamentalmente en la década de los 80 y 90, se realizaron numerosas intervenciones de eliminación de su- perficies históricas para su posterior refacción, considerándolas como actuaciones de limpieza previa. A medida que el criterio de conservación de la materia histórica estuvo más presente, fueron también más habituales las intervenciones de consolidación y limpieza de las superficies (aprox. el 45\% de las intervenciones de la última década), realizando mayoritariamente trabajos de reintegración de lagunas (27\% de las intervenciones) (Gráfico C1 de la Figura 2).

\subsection{Reflexiones generales sobre los criterios de intervención}

La combinación de los diversos grados de acción (no intervención, limpieza/consolidación, reintegración, reconstrucción) estuvo generalmente vinculada a los criterios propios del autor y a los objetivos que se pretendían conseguir con el proyecto (nuevo uso del edificio, recuperación de volúmenes, consolidación de la ruina, etc.).

En lo que atañe a los principios generales de la teoría de la restauración, se persiguió frecuentemente el principio de distinguibilidad durante todo el periodo analizado, con actua-

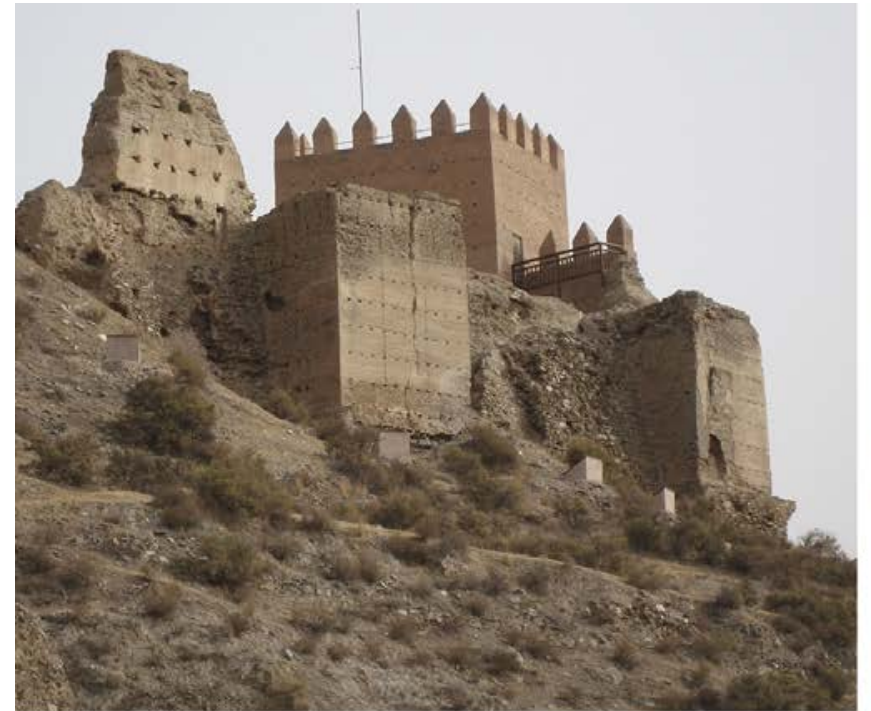

A. Estado actual del Castillo de Tabernas en la que se aprecia la intervención de 1980.

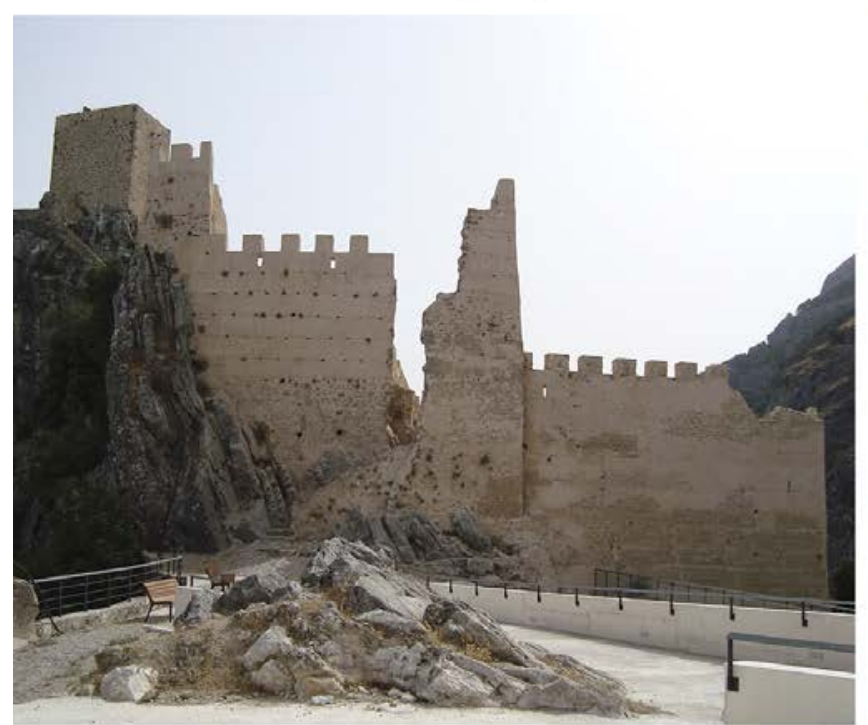

C. Estado actual de la muralla oeste del Castillo de la Iruela tras la intervención de 2006.

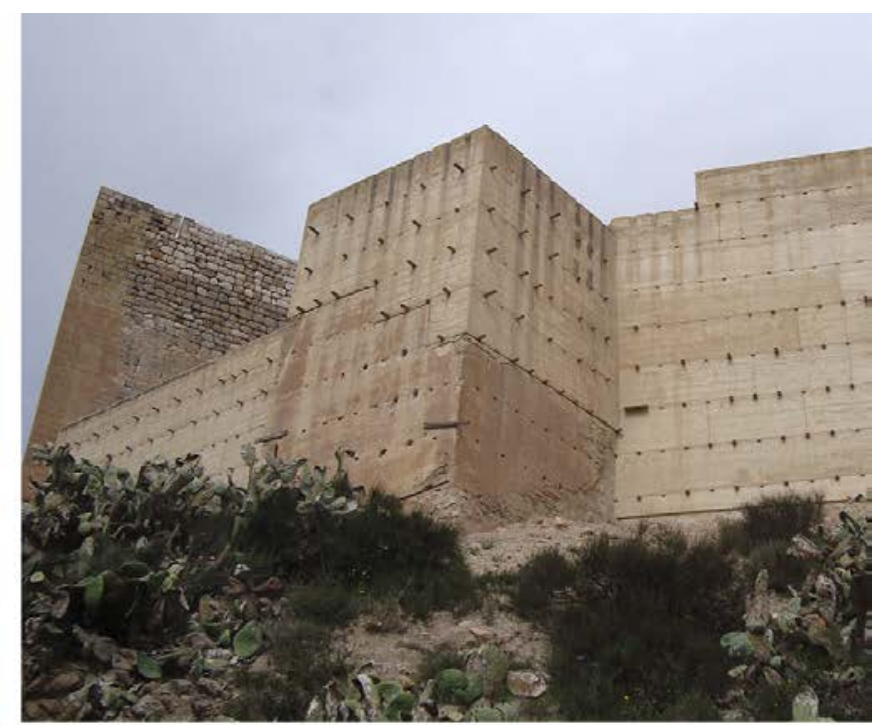

B. Estado actual del Castillo de La Mola tras la restauración de los años 90.

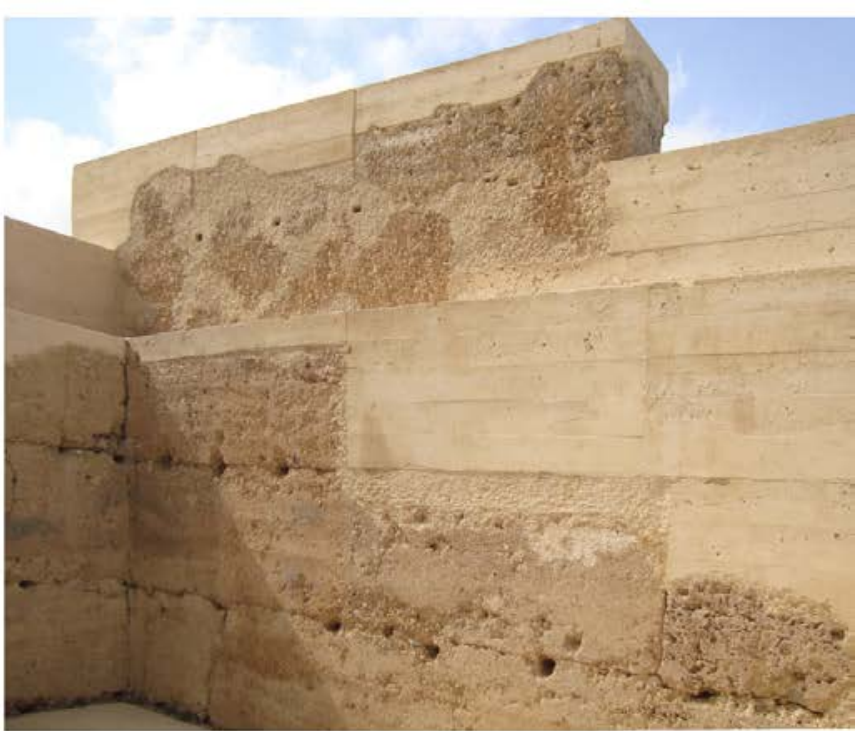

D. Estado actual del Castillo de Carrícola en la que se aprecia la intervención de 2008.

Figura 3. Imágenes de intervenciones tipo (criterios más frecuentes) de las distintas décadas analizadas. (Imagen: L. García Soriano). 
ciones en las que se buscaba la distinción clara de la nueva construcción respecto de la original, no obstante se buscara una armonía principalmente constructiva y cromática entre ambas. Además, en las más recientes se trabajan las superficies, realizándose propuestas de reintegración de lagunas en las que el material aportado queda rehundido respecto de la preexistencia.

La reversibilidad y la mínima intervención son principios a los que no se hacía apenas referencia en los proyectos más antiguos. En cambio, en los proyectos de finales de la década de 1990 y 2000 fueron frecuentes las reflexiones en torno a estos dos parámetros, a pesar de que en numerosas ocasiones no se alcanzaron satisfactoriamente. La reversibilidad fue quizás el principio menos conseguido, tanto en las intervenciones más antiguas como en las más contemporáneas.

El análisis global ha permitido observar que el panorama de los criterios de intervención que han guiado estas actuaciones ha ido cambiando con el paso del tiempo, desde las primeras propuestas con criterios más restauradores, cediendo el paso paulatinamente a criterios conservadores, reduciéndose las reconstrucciones (Figura 3). No obstante hoy en día estos criterios se inclinen claramente hacia los principios conservadores, el debate respecto a los criterios de intervención a adoptar sigue abierto en numerosos frentes, y son los criterios propios, la formación personal y la sensibilidad de cada técnico con capacidad para intervenir en el patrimonio, los que provocan que la propuesta de intervención se posicione más cerca de una línea de actuación o de otra.

\section{ANÁlISIS DE LAS TÉCNICAS CONSTRUCTIVAS PROPUESTAS}

Las técnicas constructivas propuestas en las intervenciones están íntimamente ligadas al menos a dos factores: la variante de la técnica constructiva original (vinculada generalmente con la tipología del edificio) (11) y los criterios de intervención propios de cada técnico. Por otra parte, no se debe olvidar que estas intervenciones responden, o al menos tienen la voluntad de responder, a la degradación presente en cada caso, por lo que en general se tratará de adaptar la solución a los problemas, proponiendo un tipo de solución u otro a tenor de su gravedad.

El criterio de intervención común en los años 80 de la búsqueda de una armonía estética entre lo nuevo y lo antiguo promovió la utilización de la técnica constructiva original de la tapia en la mayoría de los casos, no obstante una minoría de ellos empleó otras técnicas para realizar las reintegraciones, como los retacados con mampostería o ladrillo, propuestas heredadas de los criterios más comunes en décadas anteriores. Además, en este momento se están llevando a cabo algunas intervenciones importantes en edificios de tapia utilizando la técnica constructiva original, como es el caso de las primeras fases de restauración proyectadas por Ismael Guarner en la Muralla de Niebla (12), que servirán como referente a muchos otros proyectos contemporáneos, por ser una intervención pionera en la restauración de estas estructuras.

No obstante, a pesar de este interés y voluntad por parte de los arquitectos restauradores de emplear la técnica constructiva original, con la voluntad de mejorar la calidad de los muros, se recurre a la incorporación de materiales actuales a la masa como el cemento (que en ocasiones también se emplea en la junta entre cajones de tapia), posiblemente por ser la tapia entonces una técnica aún en ocasiones desconocida o poco explorada. Incluso en ocasiones, las actuaciones de reconstrucción volumétrica o de recuperación de la masa se realizan con fábricas de hormigón. Al realizarse estos hormigones con un encofrado similar al de las tapias tradicionales, estos muros tienen cierta similitud en su puesta en obra y acabado con los originales. Sin embargo, el empleo del cemento resta permeabilidad a estos muros y reduce considerablemente su compatibilidad material con el muro original, generando toda una serie de fenómenos de degradación asociados. Por ello, el empleo de cemento en la masa se fue reduciendo paulatinamente hasta nuestros días.

\subsection{Técnicas de intervención en los muros}

Cuando los muros están muy degradados y han perdido parte de su sección, las intervenciones propusieron generalmente adosar una nueva tapia al muro preexistente, y el encuentro entre ambos se resolvió con diversas soluciones, generalmente mecanismos de unión de la propia masa (cajeados en cola de milano) (Esquema 1 de la Figura 4), y elementos auxiliares de conexión (varillas, llaves...) o aumento de superficie de rozamiento (mallas, telas de gallinero...). Estos elementos de unión entre el material original y el de nueva aportación se resuelven principalmente con materiales contemporáneos.

Tras el análisis de los casos de estudio se puede afirmar que, a partir de finales de la década de los años 80 , se ha visto un incremento progresivo en el empleo de los materiales tradicionales (tierra, arena, grava, mampuesto, cal) en la masa de las nuevas tapias, reduciéndose generalmente el contenido en cemento, aunque este seguirá presente en numerosas intervenciones durante la década de los 90.

En esta década la tendencia general sigue siendo el empleo de la técnica constructiva original, teniendo ya mucha más sensibilidad con la misma, intentando adaptar los encofrados a la modulación existente. La incorporación del cemento en la masa de las nuevas tapias sigue realizándose de forma bastante frecuente, aunque generalmente en proporciones menores, y los elementos de unión entre el material original y el nuevo suelen resolverse también, como anteriormente, con varillas de acero. En este periodo se opta por el acero galvanizado o inoxidable con la intención de mejorar el comportamiento del acero en contacto con la masa de tierra (Esquema 3 de la Figura 4). Otra tendencia en estos proyectos es mejorar el agarre del nuevo material con el picado de la superficie existente o el cajeado de la misma, que poco a poco tenderá también a reducirse, a medida que se incrementa el criterio del respeto por el material tradicional.

El paso del tiempo ha dado a conocer los aciertos y desaciertos de estas intervenciones y esto ha influido en las propuestas de intervención más actuales, que han ido buscando mejorar las soluciones técnicas y el respeto por la preexistencia. Por ello, en la primera década del siglo XXI, se continuó revalorizando el empleo de la técnica constructiva tradicional como la opción más apropiada, y aunque persistió el empleo ocasional del cemento blanco en la masa, empezaron a surgir otros proyectos que utilizaron los materiales originales, sin añadidos. Este cambio fue fruto del creciente estudio y conocimiento de la tapia en las últimas décadas (13) (14), que permitió a los profesionales el empleo de la técnica con más rigor, obser- 


\section{1}

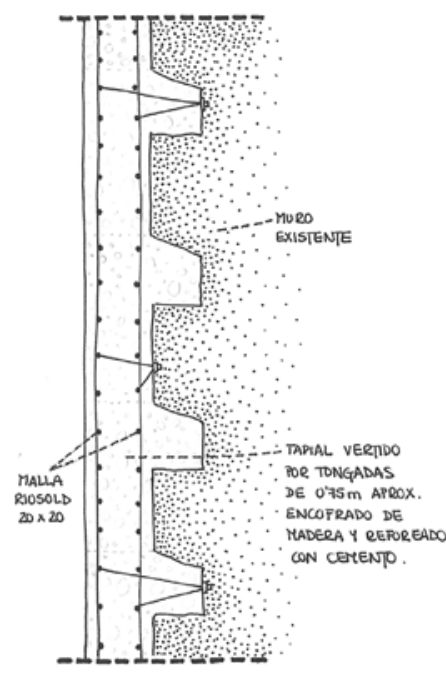

Esquema de intervención con nuevas tapias ancladas al muro con colas de milano, elaborado a partir de los detalles
consultados en el expediente PI 0042.05. (Intervención de 1984)

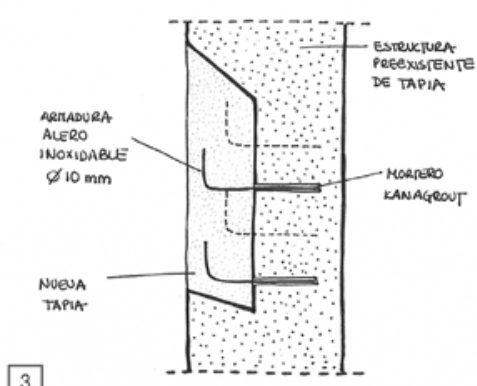

Detalle de la ejecución de nuevas tapias adosadas a la preexistencia con la incorporación de anclajes de varillas de acero inoxidable, elaborado a partir de los detalles incorporados en el expediente A4.002.18. (Intervención de 1994)

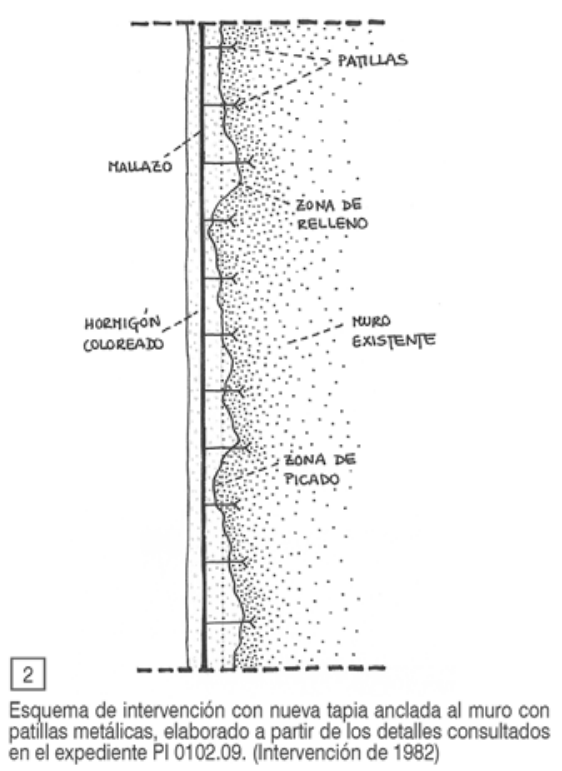

4

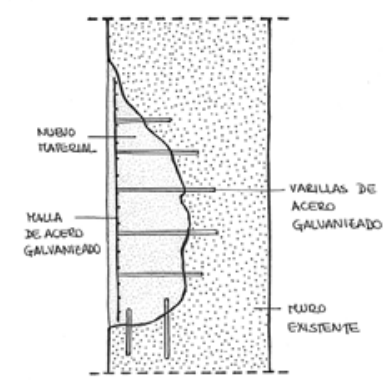

Detalle de la solución propuesta para la unión del nuevo material con el original, elaborado a partir de la documentación del expediente 10-06110-01440-06. (Intervención de 2006)

\section{5}

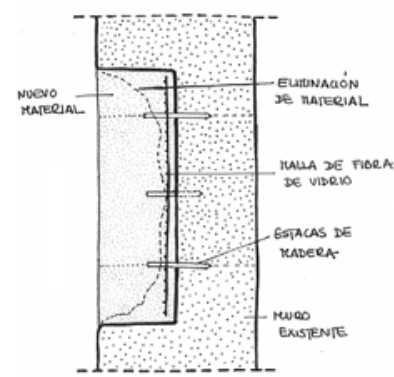

Detalle de la solución propuesta para la unión del nuevo material con el original, elaborado a partir de la documentación de expediente 01-29031-01878-09. (Intervención de 2009)

\section{7}

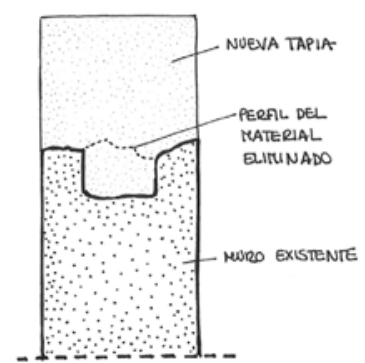

Esquema de la solución de protección de la coronación propuesta elaborado a partir de la documentación de

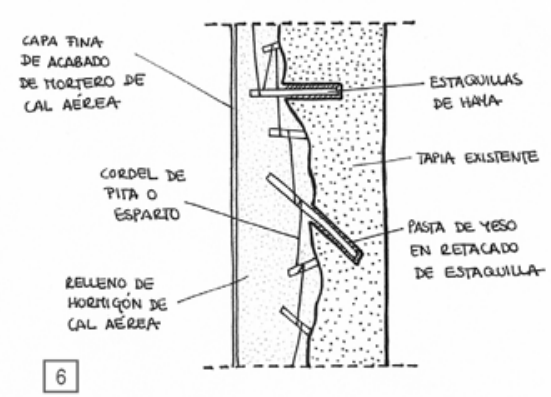

Detalle de la solución propuesta para la unión del nuevo material con el original con materiales tradicionales. Detalle elaborado a partir de la documentación del artículo "La torre de los secretos constructiva" en Loggia 24-25. (Intervención de 2008)

\section{8}

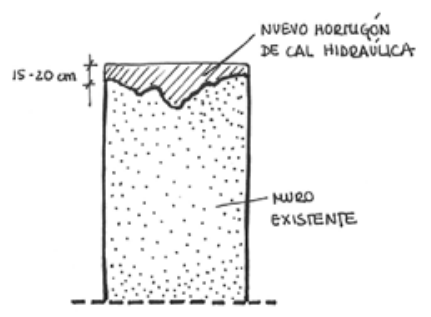

Esquema de la solución de protección de la coronación propuesta elaborado a partir de la documentacior

FIgura 4. Esquemas de distintos detalles constructivos propuestos en para la unión del nuevo material con la preexistencia. (Imagen: L. García Soriano). 
vando además que el cemento en la masa en muchos casos era fuente de degradación en el muro, como la aparición de eflorescencias que, en estado avanzado, causaban pérdidas de material importantes. La adherencia y conexión entre el material existente y el de nueva aportación fue uno de los principales problemas a resolver. Las propuestas más frecuentes propusieron la mejora del agarre aumentando la superficie de rozamiento con elementos a modo de varillas o conectores. Existen dos tipos de propuestas: las más habituales que introducen materiales contemporáneos (fundamentalmente la fibra de vidrio) (Esquema 5 de la Figura 4) y, más recientemente, las que recurren sistemáticamente a materiales tradicionales. Una línea de actuación actual propone el empleo de estacas de madera para ejecutar esta unión (Esquema 6 de la Figura 4). Siendo la madera un material originalmente empleado en la ejecución de las agujas del tapial, se tiene un cierto grado de certeza de que se trata de un material compatible con la tierra del muro.

De esta reflexión se puede concluir que el paso del tiempo ha llevado a los técnicos a experimentar con diferentes materiales para realizar esta unión, generalmente confiando en los materiales más actuales. En los años 80 fueron las barras de acero liso, en los años 90, de acero inoxidable o galvanizado, y finalmente, en la última década, las barras de fibra de vidrio y resinas. Sin embargo, las tendencias actuales vuelven la mirada al pasado y buscan en los materiales tradicionales (madera) una respuesta posible a esta unión (Figura 4).

\subsection{Técnicas de intervención en las coronaciones}

La coronación de los muros merece un trato específico por ser un punto conflictivo. En los casos analizados se detectan dos líneas de actuación en las coronaciones: bien se construyen nuevas tapias sobreelevando los muros que sirven de protección a los restos originales (Esquema 7 de la Figura 4), bien se realiza una nueva protección de la coronación con morteros similares a los empleados en las tapias, generalmente sin elevar los muros y respetando el perfil existente (Esquema 8 de la Figura 4).

En los proyectos con reconstrucción parcial o total de los muros, se opta por elevar la coronación con nuevas tapias, generalmente de forma similar a las tapias de reintegración de los muros, es decir, empleando el sistema constructivo original con la incorporación del cemento en la masa.

En los muros que poseen una cubierta (generalmente en el grupo de la arquitectura civil y religiosa), el encuentro entre la cubierta y el muro es generalmente una zona conflictiva donde pueden verificarse lesiones importantes (empujes, grietas, desplomes, falta de estanqueidad). En los proyectos más antiguos, generalmente se confía la solución a la inserción de un zuncho perimetral de atado, generalmente con los materiales modernos de la época, como el hormigón y el acero, acompañada de intervenciones de reparación de la cubierta existente.

En actuaciones más recientes, prevalece la consolidación con una capa protectora de mortero ejecutado con materiales similares a los empleados en la tapia, que respeta el perfil de los restos existentes, generalmente aplicados sobre un elemento que mejore el agarre (mallas de fibra de vidrio...). En ocasiones, esta actuación se extiende por la superficie erosionada hasta cubrir parcial o totalmente la fábrica, variando sustan- cialmente la percepción del muro histórico, no obstante su voluntad de preservar los restos.

\subsection{Técnicas de intervención en las superficies}

Durante la primera mitad de la década de los 80 una actuación generalizada en las superficies consistió en la eliminación de los restos existentes en mal estado y en la refacción extensiva, normalmente con morteros de cemento que, con el tiempo, han mostrado su incompatibilidad con la fábrica histórica de tierra, impidiéndole la transpirabilidad. A medida que las intervenciones fueron más respetuosas con la materia existente se empezaron a proponer la reintegración y consolidación de las superficies, normalmente asociadas a actuaciones previas de limpieza. Los materiales empleados en estas actuaciones siguen una tendencia similar: en las actuaciones más antiguas eran de cemento, posteriormente se han realizado con mortero bastardo y, progresivamente, el cemento ha ido desapareciendo de estos enlucidos, sustituyéndose por materiales más compatibles como la cal.

\section{RESULTADOS OBTENIDOS TRAS LAS INTERVENCIONES}

El estudio de los fenómenos de degradación causados por las propias intervenciones ha desempeñado un rol fundamental en el aprendizaje de las experiencias pasadas, puesto que el desacierto de algunas de ellas ha generado nuevas lesiones y daños, o incluso ha incrementado los existentes. Estas lecciones a extraer de las intervenciones realizadas son válidas con independencia de compartir o no los criterios de la intervención y su resultado estético.

\subsection{Resultados en los muros}

Las intervenciones generalmente de recalce en la base de los muros ejecutadas con hormigón en masa, resuelven frecuentemente los problemas estructurales, pero generan en ocasiones incompatibilidad material y fenómenos de degradación asociados, como la aparición de eflorescencias (Imagen A de la Figura 5). Las intervenciones de reconstrucción con tapia o con mampostería con el concurso del cemento poseen el mismo problema. Al margen de la técnica constructiva elegida para la reconstitución de la base del muro, el empleo de materiales más transpirables y una correcta canalización de las aguas favorecerán la durabilidad de la solución.

\subsection{Resultados en la coronación}

Los fenómenos de degradación tras las intervenciones de coronación están generalmente degradación propios del material añadido. En la superposición de nuevas tapias, el problema reside la eventual incompatibilidad entre el material original tierra y la aportación de cemento que, por su contenido en sales, puede generar la aparición de eflorescencias. Otro fenómeno de degradación frecuente deriva de la inserción de elementos metálicos que, con el tiempo, se oxidan rompiendo la fábrica y provocando desconchados y grietas. Por otro lado, en las intervenciones en las que se propone una nueva protección, los fenómenos de degradación están ligados al paso del tiempo y al envejecimiento natural del nuevo material. Si en la coronación del muro se emplean anclajes, redes o elementos de diverso tipo embutidos en la masa, se debe tener en cuenta que con la erosión de las ca- 
pas superficiales estos elementos irán aflorando (Imagen B de la Figura 5). Otro ejemplo podría ser la reconstrucción del volumen del muro una fábrica de ladrillo hueco enlucida con un mortero de cemento o de cal, estrato que con el tiempo se erosiona, desvelando la fábrica inferior que normalmente no ha sido pensada para quedar vista.

\section{5•3. Resultados en la superficie}

Estas intervenciones, que en algunos casos aumentan en espesor hasta alcanzar verdaderas reconstituciones del muro, con tapia encofrada, retacados de mampostería u hormigón en masa, generan exactamente los mismos problemas de incompatibilidad material descritos. Sin embargo, la reconstrucción del muro en su cara superficial con tapia lleva además asociada una reflexión importante sobre los anclajes. Si no se realizan mecanismos de conexión, las experiencias han mostrado que la cara añadida se desprende del muro original. Por otra parte, el empleo de elementos metálicos de conexión ha generado otros fenómenos de degradación, fundamentalmente causados por la corrosión, movimientos diferenciales, etc., como el desprendimiento de parte de las superficies, dejando al descubierto los elementos metálicos (Imagen $\mathrm{C}$ de la Figura 5). Hasta la fecha, los anclajes de madera o cuerdas de fibras naturales han demostrado ser los que mejor responden en estas intervenciones.

Por último, estas reflexiones se pueden trasladar también a la intervención en las superficies de acabado limitadas a la costra superficial o al enfoscado, donde los morteros de cal natural o mezclas de cal, tierra y arena han respondido mejor por su compatibilidad con el material existente, que los revestimientos de morteros de cemento por su menor aptitud para transpirar.

Otro fenómeno frecuente es el depósito de suciedad en las superficies generada en muchas ocasiones por no haber resuelto la coronación de los muros correctamente. Si no se estudia bien la evacuación de la coronación, los proyectos de consolidación de la cresta degradada de los restos existentes pueden favorecer la escorrentía del agua por las superficies, generando una serie de manchas de suciedad que en algunos

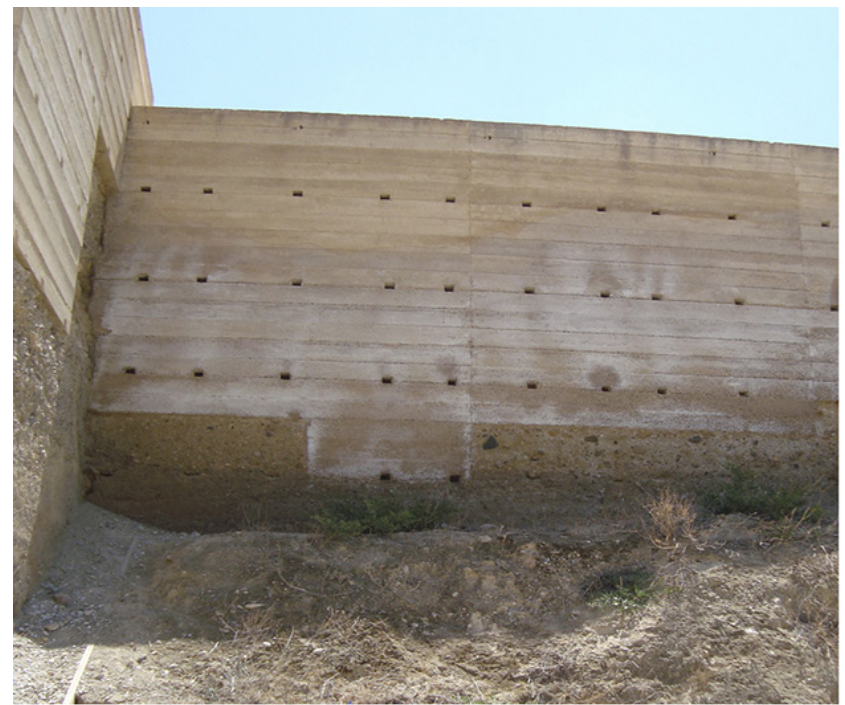

A. Estado actual del Castillo de Nogalte en la que se aprecian las manchas y eflorescencias de sales propucidas por el agua que asciende por capilaridad.

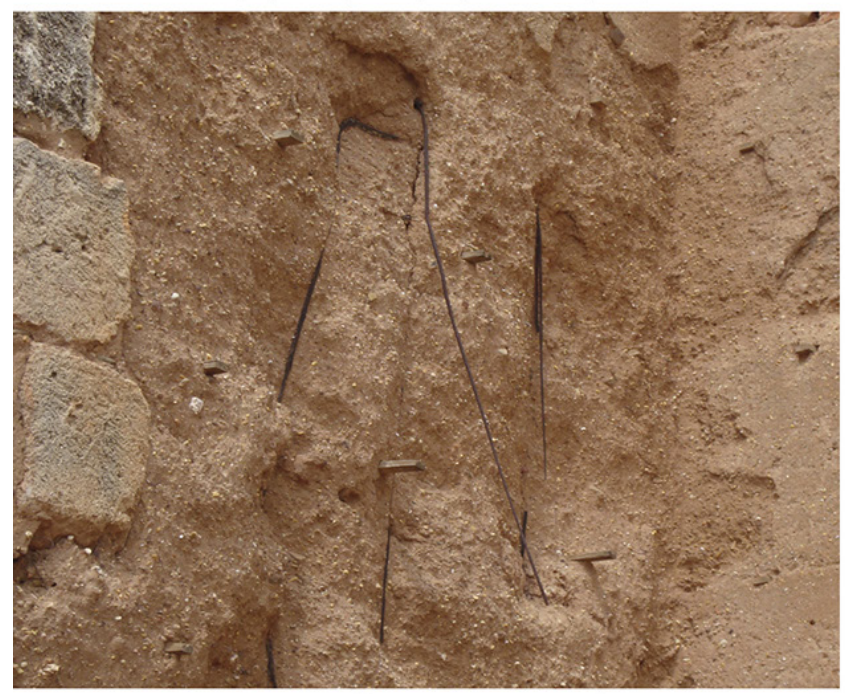

C. Estado actual de la Muralla de Niebla, en la que se aprecia que se han producido desprendimientos superficiales

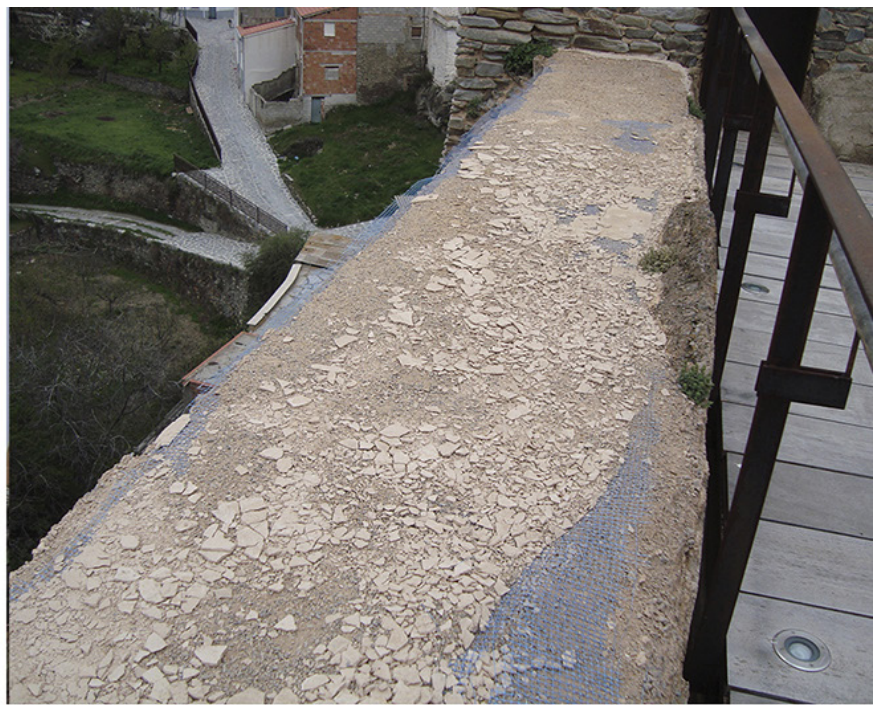

B. Imagen actual de las coronoaciones del Castillo de Bacares, en la que se aprecia la degradación de la misma que ha dejado vista la malla de unión.

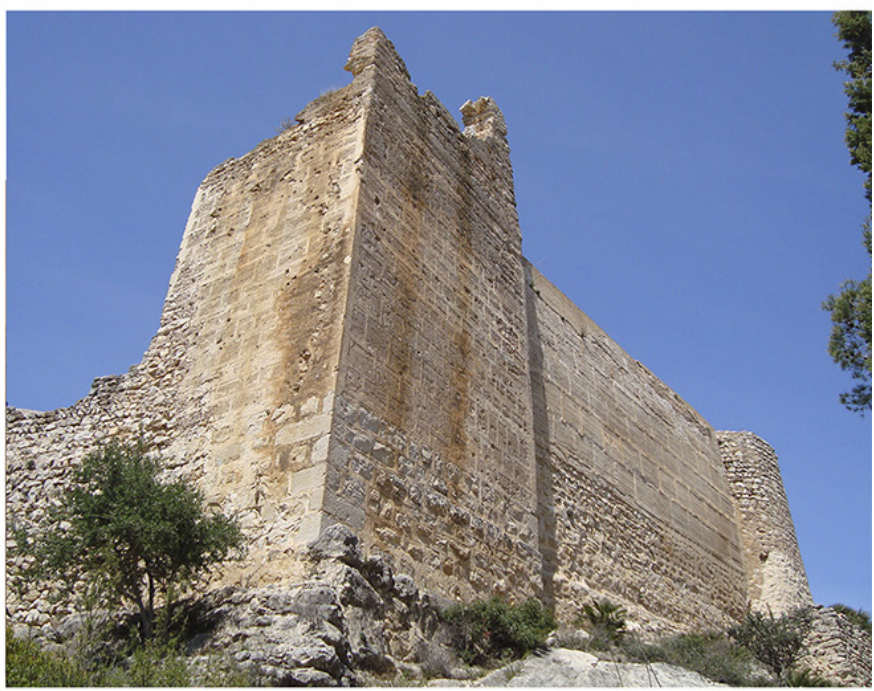

D. Estado actual Imagen actual del Castillo de Alcalá de Xivert en la que se aprecian las manchas de suciedad producidas por las escorrentías superficiales de agua desde la coronación.

Figura 5. Imágenes de diversas degradaciones producidas por las intervenciones. (Imagen: L. García Soriano). 
casos pueden llegar a generar problemas graves debidos a la erosión (Imagen D de la Figura 5).

\section{CONCLUSIONES}

El progresivo interés por el estudio de la técnica constructiva tradicional de la tapia que se detecta a partir de finales del siglo XX ha contribuido sin duda a una mayor comprensión del sistema constructivo y a una sensibilización hacia el patrimonio construido con esta técnica (16) (17) (18). Este creciente conocimiento ligado a una mayor conciencia patrimonial y a una mayor experimentación en materiales y obras caracteriza el periodo de intervenciones que ha abarcado este trabajo de investigación.

Tras el desarrollo de este trabajo, se ha podido detectar un progresivo empleo de las técnicas tradicionales tanto en la ejecución de nuevos muros como en las intervenciones de reposición de superficies. El empleo de la misma técnica constructiva se evidencia desde las primeras intervenciones de los años 80, pero es quizá en los materiales donde se ha avanzado más en estos años, reduciéndose progresivamente el empleo de materiales contemporáneos como el cemento, y quedando patente en la mayoría de los casos recientes la necesidad de trabajar con materiales tradicionales compatibles con los preexistentes.

Asimismo, cabe señalar que a lo largo del periodo estudiado ha ido aumentando no sólo la preocupación por intervenir de una manera más compatible a nivel material y estructural, sino también ha aumentado el respeto por la materia del edificio y las huellas de su historia. Cada vez son más los casos de intervenciones que tratan de conservar la materia original completando las lagunas sin pretender alcanzar el aspecto unitario de la arquitectura recién construida. $\mathrm{Al}$ mismo tiempo, se detecta un esfuerzo cada vez mayor por reducir el impacto de la fábrica de nueva construcción. La búsqueda de una mayor integración de los añadidos en el contexto de la fábrica antigua a través de la investigación en los colores de las tierras y los áridos o el tratamiento de las texturas superficiales a través de distintas técnicas como el picado, rayado, estarcido, etc. muestran una creciente conciencia de la necesidad de integrar la intervención.
Otra reflexión de este trabajo es la continuidad del paso del tiempo tras la intervención. La restauración realizada entra a formar parte del ciclo de vida del edificio y de las transformaciones materiales. Es importante tener en cuenta el factor de envejecimiento de los materiales de la intervención, no solo por su durabilidad técnica sino también por el respeto del edificio que se ha pretendido poner en valor. Es necesario por tanto tratar de evitar aquellas intervenciones que envejecen rápidamente y procurar utilizar materiales compatibles y duraderos como son los materiales tradicionales cuya durabilidad está garantizada por los siglos de su empleo. Por otra parte, es necesaria también una propuesta de mantenimiento una vez finalizada la intervención para que ésta sea más duradera al paso del tiempo.

Por otra parte, el interés desarrollado por la técnica constructiva de la tapia en las últimas décadas ha sido importante también en beneficio no sólo de la comprensión de la técnica y el uso de los materiales tradicionales, sino de su recuperación (19) (20) (21) y empleo en la arquitectura de nueva planta contemporánea más sostenible. El interés actual por la arquitectura respetuosa con su ambiente, erigida con materiales locales, donde la función y la sencillez priman frente otras cuestiones, ha orientado la mirada hacia el pasado. De este modo, la investigación de estas estructuras históricas de tapia y el conocimiento profundo de su construcción permitirá proponer nuevas actuaciones para su conservación y para el diseño y puesta en obra de una nueva arquitectura contemporánea.

\section{NOTA}

Esta investigación se ha iniciado en el marco del proyecto "La restauración de la arquitectura de tapia en la Península Ibérica. Criterios, técnicas, resultados y perspectivas" (BIA201018921; investigadora principal: Camilla Mileto) financiado por el Ministerio de Ciencia e Innovación (2011-2013) y se ha seguido desarrollando en el marco del proyecto de investigación financiado por el Ministerio de Ciencia e Innovación "La restauración y rehabilitación de arquitectura tradicional de tierra en la Península Ibérica. Líneas guía y herramientas para una intervención sostenible" (Ref.: BIA2014-55924-R; investigadores principales: Camilla Mileto y Fernando Vegas López-Manzanares).

\section{REFERENCIAS}

(1) AA.VV. (2011). Terra Europae: earthen architecture in the European Union. Pisa: ETS.

(2) Correia, M. (2007). Teoría de la conservación y su aplicación al patrimonio en tierra. En Apuntes no 20. Bogotá: Pontificia Universidad Javeriana.

(3) Mileto, C. \& Vegas, F. (Ed). (2014). La restauración de la tapia en la Península Ibérica. Criterios, técnicas, resultados y perspectivas. Valencia / Lisboa: TC Cuadernos / Argumentum.

(4) Mileto, C.; Vegas F.; Cristini, V.; García Soriano, L. (2012). Sanierung historicher Stampflehmbau-Konstruktionen auf der Iberichen Halbinsel - Kriterien, Techniken, Ergebnisse und Perspektiven / The restoration of rammed earth architecture in the Iberian Peninsula - criteria, techniques, results and perspectives. En Reinserberger J., Jörchel S. (Ed), LEHM 2012. Tagungsbeiträge del 6 Internationalen Fachtagung für Lahm-bau. Weimar: Dachverband Lehm.

(5) Carbonara, G. (1997). Avvicinamento al restauro, p. 451-510. Napoles: Liguori.

(6) Jokilehto, J. (1999). A History of Architectural Conservation, p. 295-304. Londres-New York: Elsevier.

(7) Earl, J. (2003). Building Conservation Philosophy, pp. 80-118. Dorset: Donhead.

(8) Doglioni, F. (2008). Nel restauro. Progetti per le architetture del passato, p. 85-103. Venecia: Marsilio.

(9) García Soriano, L. (2015). La restauración de la arquitectura de tapia de 1980 a la actualidad a través de los fondos del Ministerio de Cultura y del Ministerio de Fomento del Gobierno de España. Criterios, técnicas y resultados (Tesis doctoral no publicada). Valencia: Universitat Politècnica de València.

(10) AA.VV. (2007). Proyecto de restauración del castillo de La Mola. $2^{\text {a }}$ Fase. En Praxis edilicia. 10 Años con el Patrimonio Arquitectónico, p. 200-205. Valencia: Ediciones Generales de la Construcción. 
(11) Olivier, M. (1993). Restauration des estructures en terre crue en fonction de leur technologie de construction. En Conservation of Stone and other materials, vol. 2. Prevention and treatments, p. 673-680. Paris.

(12) Guarner, I. (1987). Restauración de la muralla de Niebla, Huelva. En Monografía $n^{0} 385 / 386$. Madrid: Inst. Eduardo Torroja.

(13) Maldonado Ramos, L., Castilla Pascual, F. J. \& Vela Cossío, F. (1997). La técnica del tapial en la comunidad autónoma de Madrid: Aplicación de nuevos materiales para la consolidación de muros de tapia. Informes de la Construcción, 49(452): 27-38.

(14) Monjo, J. (1998). La tierra como material de construcción. Patología y técnicas de conservación. Tratado de Rehabilitación. Tomo II: Estructuras. Madrid: Ed. Munilla-Lería.

(15) López Osorio, J. M. Restauración de la Torre de los Secretos. Castillo de Baena (Córdoba). En Loggia 24-25. Valencia: Editorial UPV.

(16) Guillaud, H. (1985). Quelques recommandations pour l'entretien et la restauration du pisé. Texto Inédito. Grenoble. École d'Architecture de Grenoble, CRAterre.

(17) Hunt, R. \& Suhr, M. (2008). Old house Handbook. A practical guide to care and repair. London: ed. Frances Lincoln Limited.

(18) AA.VV. (2008). Terra Incognita. Découbrir e preserver une Europe des Architectures de tierre. Bruselas / Lisboa: Culture Lab Editions / Argumentum.

(19) Pignal, B. (2005). Terre crue: Techniques de construction et de restauration. Paris: Eyrolles.

(20) Bollini, G. (2013). Terra battuta: técnica construttiva e recupero. Linee guida per le procedure d'intervento. Milan: Edicom Edizioni.

(21) Scarato, P. (1986). Pathologie et réhabilitation du patrimoine en pisé. Modernité de la construction en terre, p. $251-262$. Paris. 